\title{
Subtype-specific CpG island shore methylation and mutation patterns in 30 breast cancer cell lines
}

\author{
Heejoon Chae', Sangseon Lee ${ }^{2}$, Kenneth P. Nephew ${ }^{3}$ and Sun Kim², $2,5^{*}$ \\ From The 27th International Conference on Genome Informatics \\ Shanghai, China. 3-5 October 2016
}

\begin{abstract}
Background: Aberrant epigenetic modifications, including DNA methylation, are key regulators of gene activity in tumorigenesis. Breast cancer is a heterogeneous disease, and large-scale analyses indicate that tumor from normal and benign tissues, as well as molecular subtypes of breast cancer, can be distinguished based on their distinct genomic, transcriptomic, and epigenomic profiles. In this study, we used affinity-based methylation sequencing data in 30 breast cancer cell lines representing functionally distinct cancer subtypes to investigate methylation and mutation patterns at the whole genome level.

Results: Our analysis revealed significant differences in $\mathrm{CpG}$ island (CpGl) shore methylation and mutation patterns among breast cancer subtypes. In particular, the basal-like B type, a highly aggressive form of the disease, displayed distinct $\mathrm{CpGl}$ shore hypomethylation patterns that were significantly associated with downstream gene regulation. We determined that mutation rates at $\mathrm{CpG}$ sites were highly correlated with DNA methylation status and observed distinct mutation rates among the breast cancer subtypes. These findings were validated by using targeted bisulfite sequencing of differentially expressed genes $(n=85)$ among the cell lines.
\end{abstract}

Conclusions: Our results suggest that alterations in DNA methylation play critical roles in gene regulatory process as well as cytosine substitution rates at $\mathrm{CpG}$ sites in molecular subtypes of breast cancer.

Keywords: Breast cancer, Subtype, DNA methylation, CpGl shore, Mutation

\section{Background}

Breast cancer is a diverse disease consisting of multiple different molecular subtypes, such as luminal A, luminal B, triple negative/basal-like, HER2-positive, and normal breast [1]. As these subtypes are associated with differences in clinical outcomes [2], more completely describing the precise molecular nature of breast cancer may eventually allow for "personalized" clinical decisions, translating molecular information into better treatments for patients with breast cancer [3]. In this regard, gene expression

\footnotetext{
*Correspondence: sunkim.bioinfo@snu.ac.kr

${ }^{2}$ Department of Computer Science and Engineering, Seoul National University, Seoul, Republic of Korea

${ }^{4}$ Interdisciplinary Program in Bioinformatics, Seoul National University, Seoul, Republic of Korea

Full list of author information is available at the end of the article
}

patterns have been widely used not only to identify breast cancer subtypes and but also to develop clinically useful gene signatures. Microarray-based transcriptional profiling identified 50 genes used for a classifier called PAM50 (Prosigna) [4]. The 21-gene assay Oncotype DX is predictive of breast cancer recurrence and the use of this 21-gene assay has a significant impact on treatment decisions [5].

Beyond gene expression profiling, epigenetic modifications, reversible, heritable and includes changes in DNA methylation, modification of histones and altered microRNA expression levels, have received recent attention in breast cancer subtypes [6, 7]. DNA methylation patterns in particular have been used to distinguish breast cancer phenotypes [8-13], and differentially methylated regions (DMRs) as prognostic breast cancer biomarkers (patient survival analysis) have been described $[14,15]$ 
Furthermore, based on the association of DNA methylation with altered gene expression, a number of "integrated" DNA methylation/gene expression analyses have been performed, including those by Feinberg and coworkers (2009) [16] demonstrating the importance of methylation in areas surrounding $\mathrm{CpG}$ island (CpGI) shores, Brenet et al. [17] reporting the importance of 1st exon methylation, Sproul et al. (2011) [18] on the role of aberrant CPGI methylation and transcriptional repression in breast cancer lineages, our recent reports integrating DNA methylation and gene expression in breast cancer $[19,20]$. However, a comparative analysis of DNA methylation at CpGI, CpGI promoters, and CpGI shores regions, more specifically at transcription binding site (TFBS) associated overlapped regions and their impact on gene expression in breast cancer molecular subtypes on a genome-wide level has not been reported.

Gene mutations are key events in cancer development, and recent cancer genome projects have yielded extensive comparisons of the mutational landscape in breast cancer subtypes [11] and mutations associated with clinical outcomes [21, 22]. In addition, complex relationships between mutation prevalence and transcription [23], as well as an association between DNA methylation and gene mutations $[24,25]$ have been reported. Recently, in [19], we reported that genome-wide methylation profiles were distinct among breast cancer subtypes and there were methylated sites in the promotor regions of genes that were down-regulated in a cancer subtype specifically way, suggesting that the methylated sites interfered interactions between transcription factors and the promotor genomic regions. However, this study did not report signatures of methylation in specific genomic regions for breast cancer subtypes and did not investigate relationship between DNA methylation and gene mutation rates among breast cancer molecular subtypes. By integrating methylation and mutation patterns, we demonstrated that:

1. Differential CpGI shore methylation patterns were characteristic of the basal B subtype. Furthermore, within CpGI shores, methylation at TFBSs and overlapping promoter $\mathrm{CpGI}$ regions was associated with differential gene regulation in basal B compared to other breast cancer subtypes.

2. Basal A breast cancer cells showed higher mutation rates at $\mathrm{CpG}$ sites with low or intermediate methylation, whereas mutation rates were higher at hypermethylated CpG sites in the basal B subtype.

\section{Motivation}

This work was motivated by our previous works in modeling DNA methylation susceptibility [26-28] and conservation of CpG island sequences [29]. We and many scientists believe that DNA methylation is not random and probably there is an instructive mechanisms embedded in the genomic sequences [30]. Thus our motivation is to investigate where there is any notable correlation between mutations (cancer-subtype specific genomic sequences) and cancer subtype specific methylation patterns. In fact, there is recent article that suggests associations between mutations and epigenetic changes [31]. Thus our goal in this study is to look for any association between genome sequence differences and methylation patterns.

\section{Methods}

30 breast cancer cell line and subtype difference estimation Genome wide DNA methylation status was measured in our previous work [19] by MBDCap sequencing from 30 breast cancer cell lines representing three different molecular subtypes; basal A, basal B and luminal obtained from (see Additional file 1: Supplementary Table S1 for more information on cell lines). MBDCap-seq utilizes affinity between MBD protein and methylated DNA sequence and allows cost-efficient measurement of genome wide DNA methylation status. Initial quality trimming is performed by Trim Galore [32] to remove bad sequence quality reads, and remained reads were aligned to reference genome (build hg19) by using Bowtie2 [33] with seed length 22 and allowing zero mismatch in it. Multiple and duplicated reads are then filtered out to mediate the possible PCR amplication bias. Aligned reads were counted through genome-wide scanning with 100bp length window by using MEDIPS, a R package providing fixed-length bin methylation estimation from affinity based sequencing data in the form of relative methylation score (RMS) [34]. The RMS value of each 100bp bin was then compared across the tumor subtypes to extract DMRs and their significance were tested by $\mathrm{t}$-test with adjusted $P$-value (Bonferroni) $<0.05$.

Affymetrix microarray based gene expression data was downloaded from [35] and expression level is measured by R Limma package [36] in Bioconductor. Background correction and normalization is performed on signal intensity to measure expression, and pair-wise and three classes subtype gene expression comparison was performed to extract differentially expressed genes (DEG)s. For the pairwise gene expression comparison, linear model based Limma was used, and for three class comparison, mutual information based DEGPack [37] was used.

Normal breast control data were obtained from TCGA data portal (measured by whole genome bisulfite sequencing (WGBS); id: TCGA-A7-A0CE-11A-21D-A148-05) and from genome wide methylome study [14] (measured by MBDcap sequencing). Initial quality trimming and aligning were performed on both data set, and genome wide methylation status of TCGA WGBS data and 
MBDCap sequencing data was measured by methylKit [38] and MEDIPS [34] respectively.

\section{Targeted bisulfite-treatment sequencing}

Our previous work [19] used MBDCap sequencing data without bisulfite treatment. Thus we performed targeted bisulfite sequencing on 85 gene regions. Among 30 breast cancer cell lines, six samples (two lines representing each subtypes; see Additional file 1: Supplementary Table S1) were selected for targeted bisulfite treatment sequencing validation. Pre-library preparation utilized $3 \mu \mathrm{g}$ DNA and all libraries passed a minimum fragment size of 200 to $250 \mathrm{bp}$ and $\geq 147 \mathrm{ng} / \mu \mathrm{l}$ quality control. Hybridization was performed using SureSelect ${ }^{T X}$ Methyl-Seq Kit followed by post library generation with targeted genomic region information. Final library concentration was $250>$ pM. Based on the captured library, bisulfite conversion was performed to distinguish methylated and unmethylated DNA regions.

Sequencing was performed on 85 distinct DEG regions with additional $10 \mathrm{Kbp}$ upstream of transcription start site (TSS) using Illumina HiSeq2500. A total of 300 million reads were aligned to reference genome (build hg19) with bisulfite conversion by using Bismark [39], and each CpG site methylation was measured by using methylKit [38].

\section{Correlation between targeted bisulfite-treatment sequencing and MBDcap sequencing}

Affinity based MBDcap sequencing captures methylated reads and number of mapped reads at certain range represents the methylation status on that. On the other hand, bisulfite treatment converts only un-methylated cytosine to uracil and given that information it provides methylation level in single base pair resolution. In order to estimate the correlation between methylation levels measured by BS seq and MBDcap seq, genome-wide single base pair read coverage was measured from MBDcap seq data. Then, CpG site read coverage was extracted and intersected with targeted bisulfite treated regions to filter out result from other regions. Lastly, $2 \mathrm{~kb}$ bin methylation level were computed on both methods, and Pearson's correlation was estimated between them.

\section{Experimentally validated transcription factor binding site and their methylation status}

In search of the specific transcription factor binding sites (TFBSs) located in CpGI shores and the overlapping promoter region, we utilized match algorithm from TRANSFAC [40]. Promoter sequences were extracted from $2 \mathrm{~Kb}$ upstream of the TSS in each DEGs, and TF motif weighted matrices were used to scan the TFBSs on the sequence regions. Once TFBSs were predicted, we computed the TFBS specific methylation level by averaging methylation levels in all 100 bp bins overlapping the TFBS. Finally, we adopted experimentally validated ChiP-seq databases
(HTRIdb [41], and ChEA [42]) to verify TF binding on predicted TFBSs. In order to investigate potential downstream effect caused by methylation difference on TFBS, differential methylation across tumor subtypes was measured on TFBSs by Kruskal Wallis test (FDR $<0.1)$ and correlation with downstream gene was estimated (Spearman's rho <-0.5). To remove effect of TFs on gene regulation, we considered only TFs with similar gene expression levels, allowing us to focus on the role of DNA methylation on downstream genes.

\section{Mutation rate and subtype specific mutation}

MBDcap-seq is a DNA sequencing technology capturing methylated regions by utilizing affinity between MBD protein and methylated DNA sequence. To investigate the relationship between methyl-CpG mutation and their methylation level, genome wide point mutation discovery (matches short reads to the hg19 build) was performed on MBDcap-seq data by using the mpileup algorithm in the Samtools suite (version 0.1.19) [43]. Minimum base quality for a base to be considered was set to 13 , and maximum reads per sample was set to 250 . By incorporating sequence and quality information and mismatch sharing rates across the samples, every read having mismatches with the reference genome was statistically tested to determine whether or not the observation was due to sequencing error. In order to reduce false mutation detection caused by misaligments and indel, base alignment quality (BAQ), Phred-scaled probability of a read based being misaligned, is applied to each base [44]. In addition, anomalous read pairs in variant calling were skipped. Finally, mutation rates within a certain methylation range across the tumor subtypes were computed. We defined mutation rate as the ratio of number of cytosine substitution occurrence over the number of all $\mathrm{CpG}$ sites. In order to estimate statistical significant of computed mutation rate within certain methylation range, the mutation rate information is pooled into subtypes and tested by ANOVA with Bonferroni correction. In addition to detecting variants from all samples, subtype specific mutations were also measured. Each detected mutation was checked as to whether the observation was from all samples or only certain tumor subtype samples. We defined a subtype specific mutations as those that occurred in at least 30\% of a particular subtype but in less than $10 \%$ of the other two subtypes.

Whole schematic analysis workflow is illustrated in Fig. 1.

\section{Results}

Genome wide methylation profile and differentially methylated regions

Genome wide methylation landscape was determined in 30 breast cancer cell lines MBDCap-seq. Methylation 


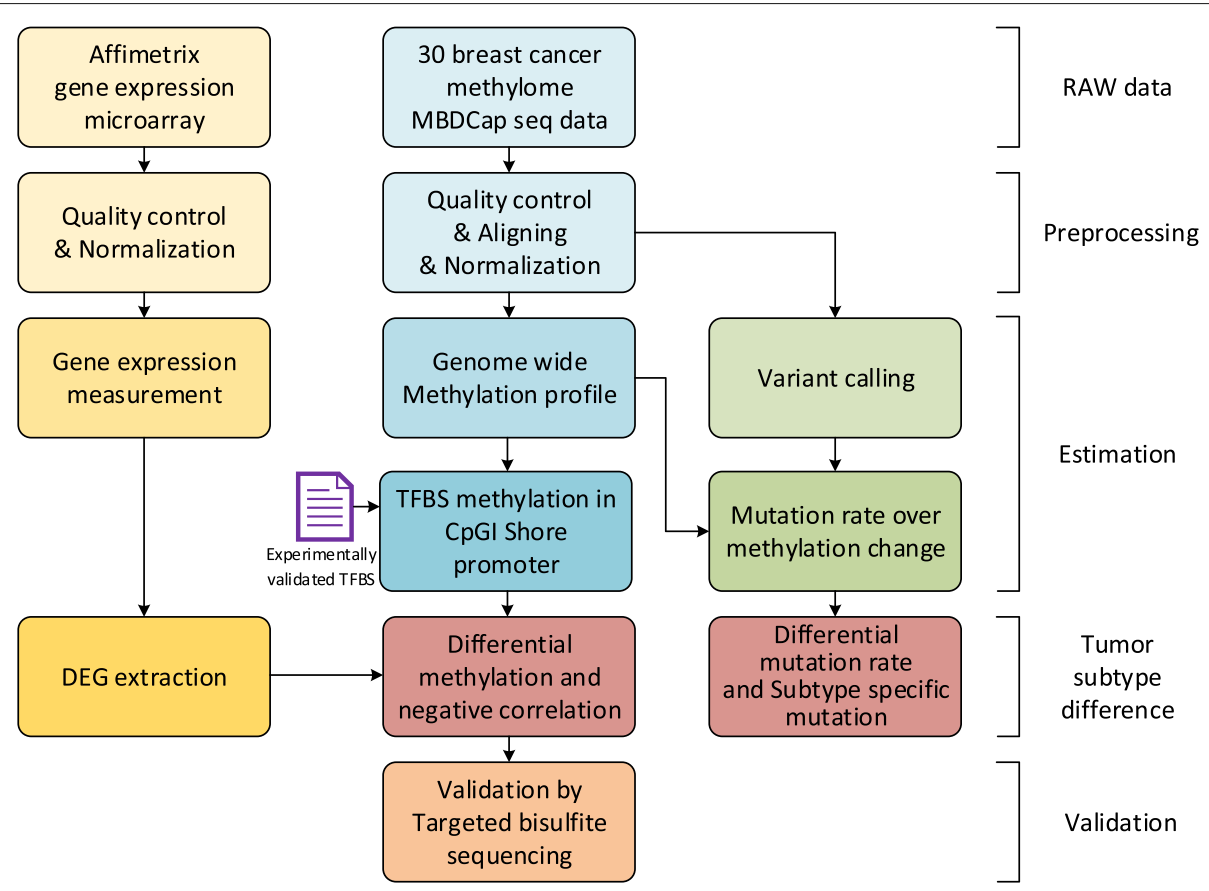

Fig. 1 Workflow for the methylation and mutation analysis of 30 breast cancer cell lines. A total of 30 breast cancer cell lines representing molecular subtypes of the disease were examined in this study. Analysis starts with quality control and normalization on both MBDcap sequencing data and Affimatrix gene expression data, and methylation and expression level were measured. During integrated analysis, subtype comparison was performed to estimate differentially expressed genes (DEG)s and differentially methylated regions (DMR)s. Experimentally validated transcription factor binding site (TFBS) information is used to estimate TFBS specific methylation level in promoter and CpGl shore overlapped region, and correlation was measured with downstream gene expression. By utilizing mutation information estimated from MBDcap sequencing, subtype specific mutation rate over methylation level was measured. Finally, single base pair resolution bisulfite treatment sequencing was performed to validated the methylation status measured by MBDcap sequencing

profiling using more than 30 million reads covered 23,149,286 CpG sites, 25,974 CpG islands, 54,543 CpGI shores, and 38,208 promoter regions $(82,91,95$, and $99 \%$ of the total in the human genome, respectively), and for overlapped regions, 10,910 promoter-CpGI and 16,227 promoter-CpGI shores (90 and $98 \%$ of total in human genome) were covered. A total 4,366 differentially methylated 100bp-bins corresponding to 2,055 differentially methylated regions (DMRs; MEDIPS package, adjusted $P$-value (Bonferroni) $<0.05$ ) were determined (see Methods). 126 DMRs were identified in the luminal and basal A pair, 1,136 in the luminal and basal B pair, and 793 in the basal A and basal B pair. Statistics of differentially methylated bins were further grouped according to the genomic regions such as $3^{\prime} \mathrm{UTR}, 5^{\prime} \mathrm{UTR}$, exon, intron, promoter, CpGI, CpGI shelf, and CpGI shore. Notably large number of differentially methylated bins were observed in intron and $\mathrm{CPGI}$ shore region from $\mathrm{Lu}-$ $\mathrm{BaB}$ pair and $\mathrm{BaA}-\mathrm{BaB}$ pair (Fig. 2a). Then, based on these comparison results, hypomethylation ratio of each subtype was further measured. In both intron and $\mathrm{CpGI}$ shore region, more than $75 \%$ (BaA-BaB pair) and $50 \%(\mathrm{Lu}-\mathrm{BaB}$ pair) of differentially methylated bins are hypomethylated in Basal B subtype (Fig. 2b). Hypomethylation ratio of other regions are in Supplementary Figure S1 (see Additional file 1).

\section{Methylation status validation by targeted bisulfite sequencing}

Affinity-based MBDCap-seq technology is a cost-efficient method to estimate genome-wide DNA methylation. However, it does not measure methylation level at the single nucleotide resolution, especially in high $\mathrm{CpG}$ density regions. In order to verify the methylation level estimated by MBDCap-seq method, we conducted targeted bisulfite-treated sequencing (BS-seq) on the genome regions around significantly differentially expressed genes (DEGs) (from 10Kbp upstream of the TSS to transcription end site (TES) including the corresponding promoter; see details in Methods). We compared methylation levels estimated from MBDcap-seq and BS-seq, and observed a strong average correlation (Pearson's correlation coefficient 0.77) and up to 0.91 between two techniques (see Additional file 1: Figure S2), demonstrating that MBDCap-seq reliably measured genome wide methylation levels. 


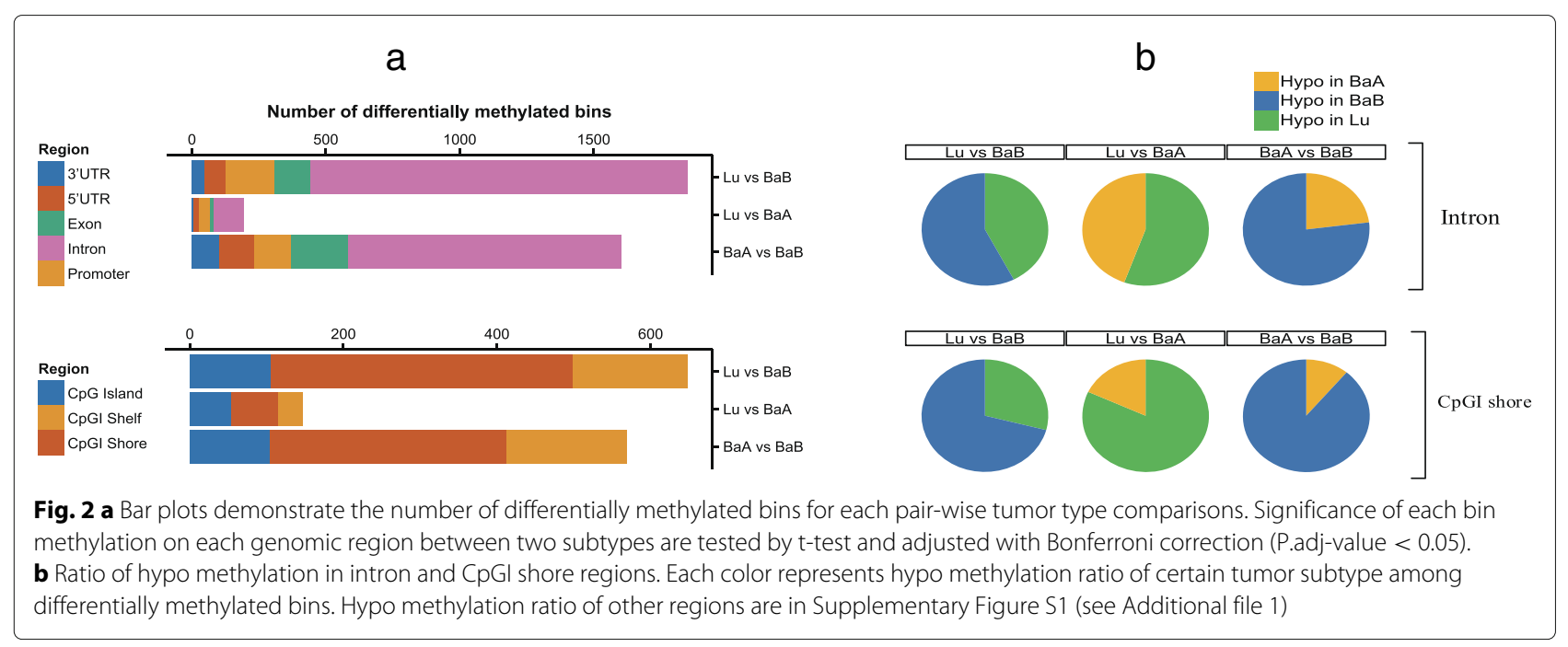

\section{Global CpGI shore hypomethylation specific to basal B tumor type}

Based on the genome-wide methylome analysis using affinity based MBDcap-seq data, we observed genome wide hypomethylation in the basal B subtype at various genomic regions. The average methylation levels in genebody, exon, as well as Dnase I hypersensitive sites were lowest in basal B (see Additional file 1: Figure S3). In addition, significant differential methylation patterns were observed in boundary areas between CPGI and CpGI shore. Notably, while methylation level peaks were observed in luminal and basal A, the steep peaks tapered into a gentle slope or nearly flattened out in basal B (Fig. 3a). In addition, from heatmap for genome wide CpGI and their flanking area, highly methylated boundary region in luminal and basal $\mathrm{A}$ are observed, but not from basal B (Fig. 3b). Significance of differential methylation among subtypes in entire $\mathrm{CpGI}$ and their flanking regions was tested by ANOVA and the $P$-value is adjusted by Bonferroni correction. From the result of statistical significance estimation, we identified that adjacent regions between $\mathrm{CpGI}$ and $\mathrm{CpGI}$ shore area have significantly low adjusted $P$-value compared to near regions (Fig. 3c).

In order to further validate the observed methylation patterns, we utilized two normal data set; TCGA normal breast data measured by WGBS and normal data from genome wide methylome analysis study [14] measured by MBDcap sequencing. Genome wide methylation level were estimated through same analysis procedure (see "Methods") and estimated average methylation in CpGI and CpGI shore regions. From methylation result based on both normal data, we observed same pattern and found steep peaks as well in adjacent region between $\mathrm{CpGI}$ and CpGI shore (Fig. 3d).

To investigate whether the differences in methylation patterns in CpGI and adjacent region CpGI shore potentially involved in gene regulation, we focused on promoter CPGI shore with transcription factor binding site (TFBS). Estimated TFBS specific methylation status (see "Methods") in the promoter CpGI shore was compared with downstream gene expression, and the TF binding to these TFBS regions was also measured to determine whether a TF influences gene regulation. That is, we investigated whether the differentially methylated TFBS in promoter CpGI shore regions among breast cancer subtypes potentially give influence to expression of downstream genes that TF regulate.

We identified 55 genes with differentially methylated promoter TFBS regions (Kruskal Wallis test, FDR < 0.1) and inversely correlated (Spearman's rho $<-0.5$ ) gene expression (see Additional file 1: Table S2). Interestingly, $55 \%$ of these genes were hypomethylated in basal B, including CAV1 and PTRF (caveolae associated protein coding genes). Epigenetic modification of these caveolae related genes was recently reported to be associated with disease [45]. Furthermore, a significant influence of CpGI shore methylation on CAV1 in breast cancer was previously reported [20]. We confirmed this finding, detecting a significant DMR within the CpGI shore overlapping the CAV1 promoter (Fig. 4). We then further investigated the methylation status of TFBS located in the CAV1 promoter and the overlapping CpGI shore region. Interestingly, the experimentally validated TFBS regions showed significant differential methylation (Kruskal Wallis test, FDR $<0.005$ ). In addition to CAV1, promoter and CpGI shore methylation with TFBS of PTRF, TGFB1, and GDF15 genes are depicted in Fig. 4. All these TFBS specific methylation within promoter and CPGI shore overlapped had inverse correlation with downstream gene expression that the TFs associated. Finally, CpGI shore methylation was validated (single base pair resolution) using targeted bisulfite sequencing (see Additional file 1: Figure S4). 


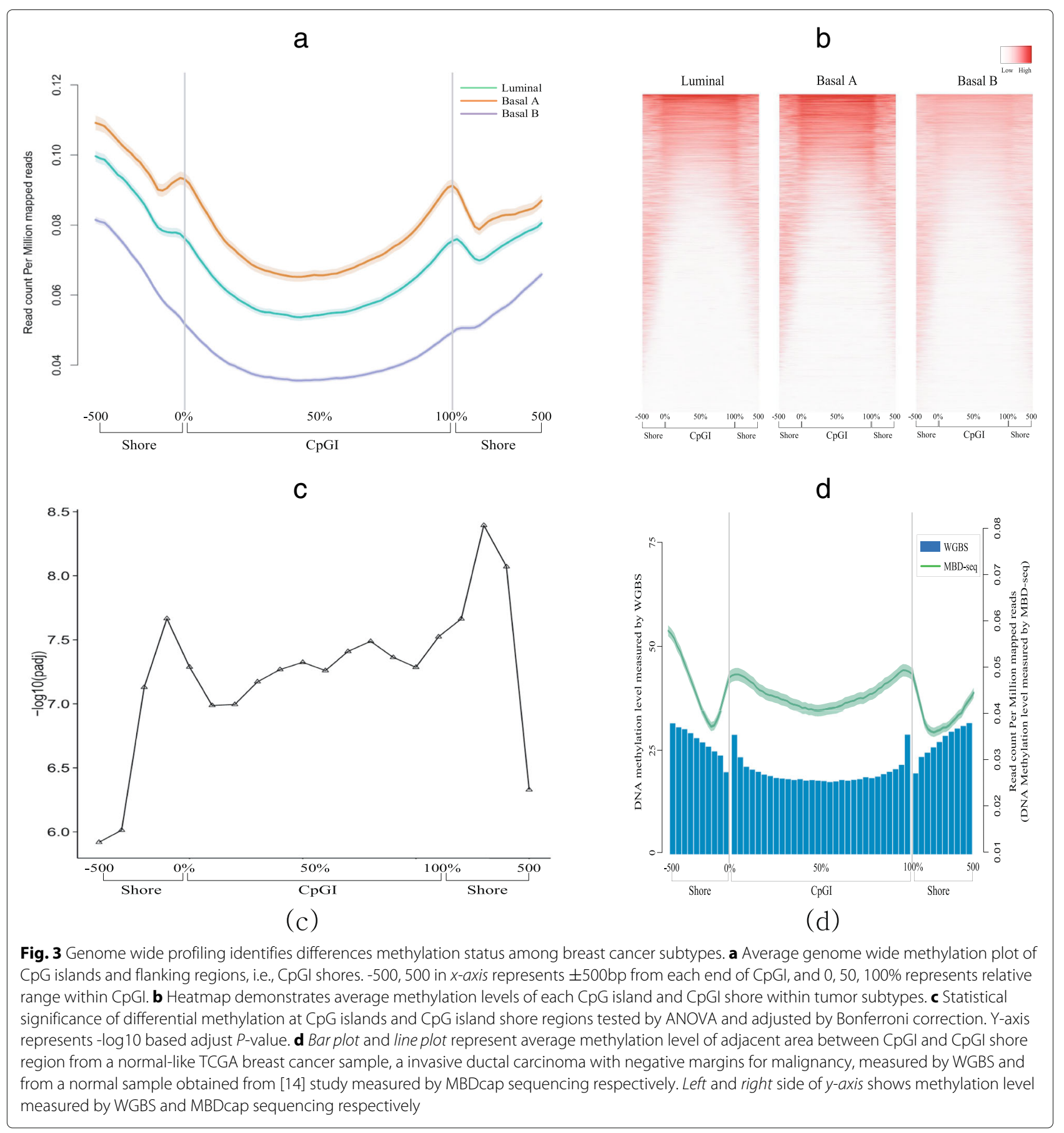

\section{Correlation between mutation and methylation across molecular tumor subtypes}

In order to investigate the relationship between mutations and methylation variation, cytosine substitution rate on CpG site was computed across the tumor subtypes. By comparing the genome wide methylation profile and estimated mutation frequencies, the mutation rate gradually changed as the methylation level increased in all samples. We then compared mutation rates across the tumor subtypes and found that the mutation rate pattern over the methylation change was significantly different in different subtypes. At CpG sites displaying low and intermediate methylation, luminal and basal B had similar mutation rate but basal A showed a distinct and higher mutation rate $\left(P\right.$-value $=1.1258 \times 10^{-2}$ by ANOVA test with Bonferroni correction). Conversely, at highly methylated CpG sites, luminal and basal A had similar mutation rates but the mutation rate was significantly different 


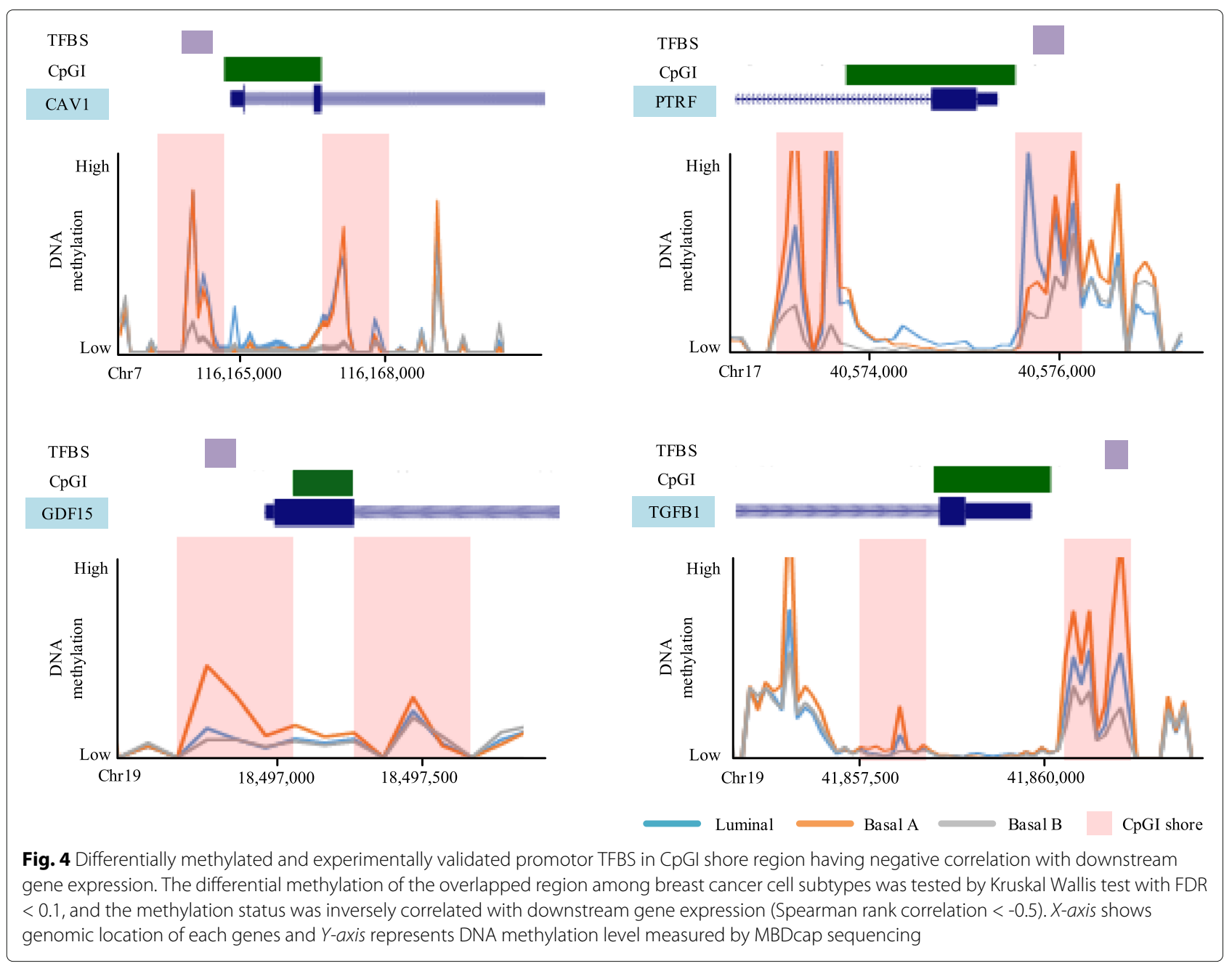

$\left(P\right.$-value $=6.84 \times 10^{-7}$ by ANOVA test with Bonferroni correction) for the basal B subtype (Fig. 5).

To find possible biological explanation of the observed mutation rate difference across the tumor subtypes, we investigated whether there were any regional genomic effects. We first divided observed mutations by various regional groups based on their genomic position information. We then extracted subtype specific mutations, a mutation that occurs frequently in one subtype but rarely observed from others, by filtering out common mutation over all subtypes in each regional group (see Methods). Interestingly, in CPGI regions (known as "methyl protected" and thus hypomethylated regions) including CPGI shore and shelf, basal A specific mutations occurred the most frequently, and CpGI shore and shelf region showed significant differential subtype specific mutation occurrence (tested by ANOVA with adjusted $P$-value (Bonferroni) < 0.05). On the other hand, basal B specific mutations were significantly more frequent in intron regions (ANOVA, P.adj (Bonferroni) < 0.05) (Fig. 6). Our analysis suggests that mutation rate difference may result from regional subtype specific mutation occurrence and their methylation difference across the subtypes.

\section{Discussion}

In this study, we report two novel findings associated with tumor subtype differences in terms of methylation and mutations. For the methylation pattern, we showed that CpGI shore methylation is a distinct signature for breast cancer subtypes and also that CpGI shore methylation is associated with subtype specific gene regulation. For the subtype specific methylation patterns, there are a number of studies. Previously, Holm et al., showed that unsupervised methylation pattern analysis could distinguish molecular subtypes [9]. Jadhav et al., reported differential methylation patterns in promoter $\mathrm{CpGI}$, intragenic and intergenic $\mathrm{CpGI}$ as well as non-CpGI promoter regions compared to normal samples [46] and Kamalakaran et al., reported differential methylation pattern and association with clinical variable in luminal subtype [47]. More recently, Stefansson et al., tried to define additional epigenetic subtypes based on differential methylation patterns 


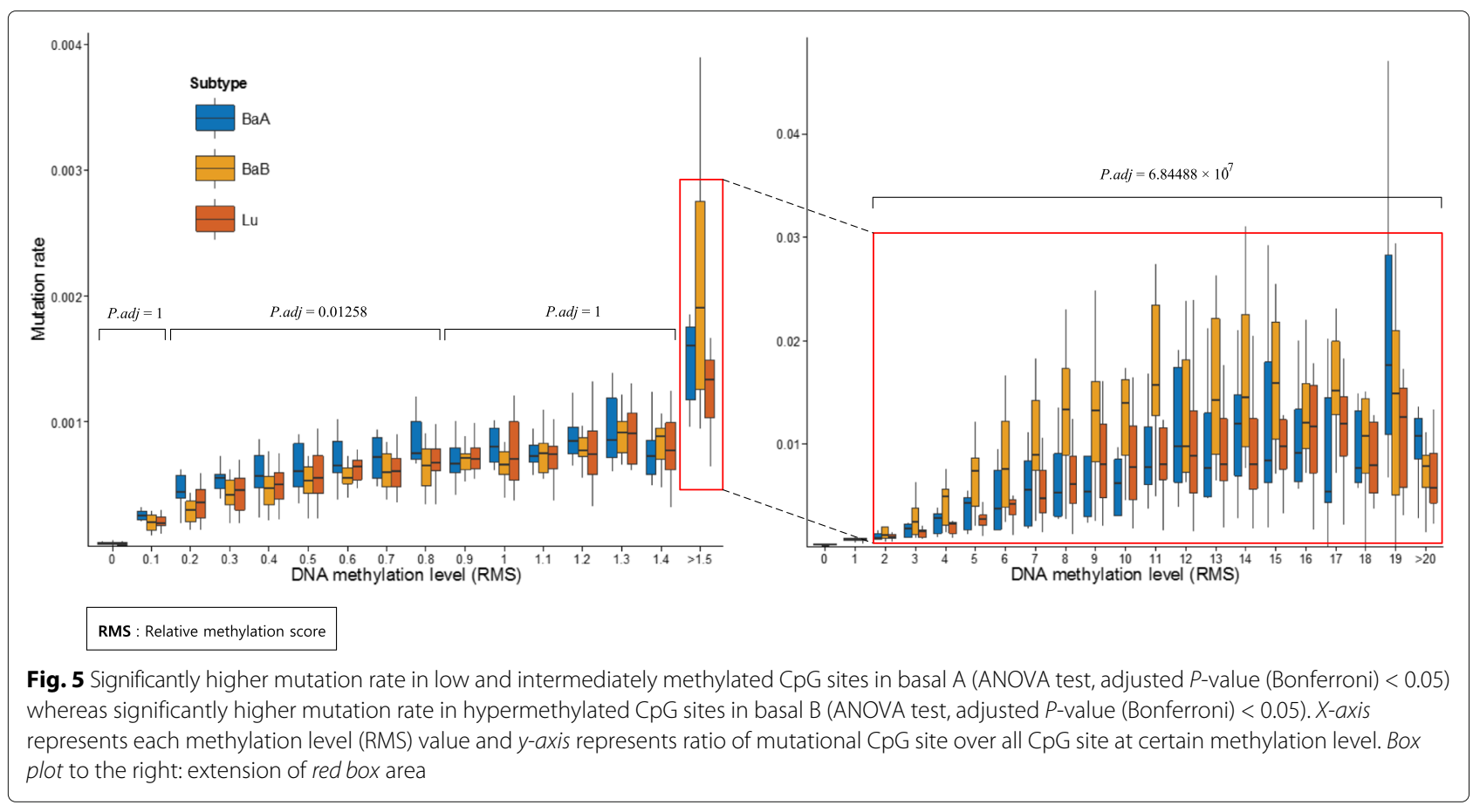

[13]. In agreement with previous studies, we observed significant differential methylation pattern on CpGI shore and promoter overlapping regions. Our further analysis on TFBS specific methylation revealed strong inverse correlation to downstream genes. We also detected more prevalent hypomethylated DMR bins in intron region for basal B subtype and this finding is in agreement with previously described genebody hypomethylation pattern studied by Yang et al. [48]. This genebody hypomethylation phenotype is also linked to hormonereceptor negative/basal-like breast cancers as described in Hon et al. [49].

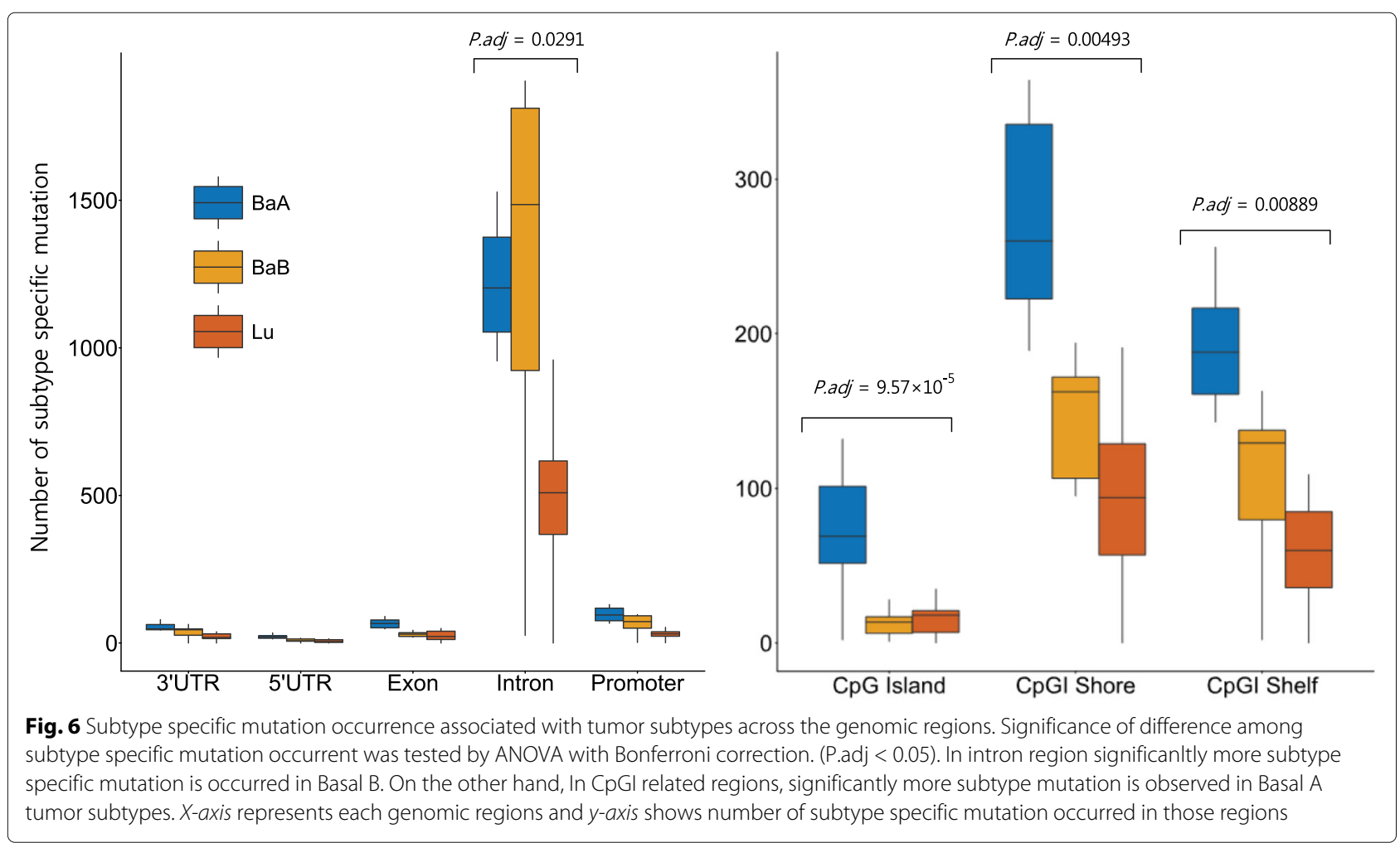


In addition to genome wide differential methylation pattern, our integrated analysis identified genes having significant differential methylation on their TFBS located in promoter CPGI shore region, and having inverse correlation with their gene expression. CAV1 and PTRF are previously reported as cancer-associated caveolae genes [20]. GDF15 and TGFB1 genes are members of transforming growth factor beta family, and encode multifunctional proteins associated with proliferation, differentiation, adhesion, and migration. Therapeutically, these genes are related to response of breast cancer cells to radiation, specifically inhibiting radiation-induced cell death and related cytotoxic action [50] and a direct association between promoter methylation and expression of these genes are reported [51]. In addition, integrated analysis showed GSTP1 and PALLD genes having low level gene expression as well as significantly higher methylation level of these gene promoters in luminal phenotype compared to the other two subtypes. Hypermethylation of the GSTP1 promoter has also been previously reported as having association with prognostic values [52], and repression of PALLD gene has been shown to contribute to invasive motility [53] and cancer cell migration [54]. Including these genes, a large number of detected genes from our analysis have overlapping of promoter regions with DHS region as well as polycom-associated H3K27me3 marked region, suggesting a potential interplay with gene transcription and that differential methylation may play important roles across the subtypes.

Mutations play an important role in the development of cancer. Several studies investigated relationship between DNA methylation and mutation. Carina et al. reported a relationship between CpG cytosine mutation rates in intron regions in human genes and variation in methylation levels as well as a positive correlation with non-CpG divergences, and a negative correlation with GC content [55]. In another study focusing on exonic regions [24], methylation in first exon regions significantly correlated with $\mathrm{C}$ to $\mathrm{T}$ substitution rate in $\mathrm{CpG}$ sites. Based on genome wide mutation rate measurements, $\mathrm{CpG}$ sites with low-to-intermediate methylation level had higher CPG substitution rates compared to other methylated $\mathrm{CpG}$ sites [25]. Our genome wide mutation rate analysis shows notable differences in mutation rates across the tumor subtypes, which correlates with methylation status. In summary, our findings on mutation and methylation indicates a trend for higher mutation rates in basal A type at low to intermediate methylation level CpG sites whereas in the basal $\mathrm{B}$ phenotype, mutation rates are higher at highly methylated CpG sites.

\section{Conclusion}

By utilizing methylome data and gene expression for 30 breast cancer cell lines, we report two novel findings.
First, our genome wide integrated analysis shows significant difference in the CpGI shore methylation pattern among breast cancer molecular subtypes. Further investigation of these regions identified 55 genes with differentially methylated promoter regions overlapping CpGI shore regions with an inverse correlation of methylation level and transcriptional regulation of these 55 genes, but no apparent difference in expression of TFs that could potentially interact with their promoter CpGI regions. This consideration of TF and TFBS provides strong evidence for the suppressive role of DNA methylation on the downstream genes. Second, we found a genome-wide relationships between mutation rate and methylation level in the molecular subtypes. From the integrated analysis, we report that mutation rate gradually increases as methylation level increases. We further investigated this pattern in relation with the molecular subtypes and found higher mutation rates in basal A when the methylation level is low-to-intermediate, but basal B breast cancer cells have higher mutation rates when the methylation level is high. We believe our findings addresses a timely issue regarding the relation between DNA methylation and mutation in terms of gene expression in tumorigenesis.

\section{Additional file}

Additional file 1: Supplementary file contains Supplementary Figure S1-4 and Table S1-2. (PDF $2170 \mathrm{~kb}$ )

\section{Acknowledgments}

Not applicable.

\section{Declarations}

This article has been published as part of BMC Systems Biology Volume 10 Supplement 4, 2016: Proceedings of the 27th International Conference on Genome Informatics: systems biology. The full contents of the supplement are available online at http://bmcsystbiol.biomedcentral.com/articles/ supplements/volume-10-supplement-4.

\section{Funding}

This research was supported by a grant of the Korea Health Technology R\&D Project through the Korea Health Industry Development Institute (KHIDI), funded by the Ministry of Health \& Welfare, Republic of Korea (grant number : HI15C3224), by Collaborative Genome Program for Fostering New

Post-Genome industry through the National Research Foundation of Korea (NRF) funded by the Ministry of Science ICT and Future Planning (2014M3C9A3063541), by the Bio \& Medical Technology Development Program of the NRF funded by the Ministry of Science, ICT \& Future Planning (2012M3A9D1054622), and funding from the Integrated Cancer Biology Program of the National Cancer Institute (Awards CA13001). Publication charges for this article have been funded by a grant of the KHIDI, funded by the Ministry of Health \& Welfare, Republic of Korea (grant number : HI15C3224).

\section{Availability of data and materials} Not applicable.

\section{Authors' contributions}

SK conceived the experiment, SK and HC conducted the experiment, HC drafted the manuscript, HC and SL processed data and analyzed results, KN prepared samples and reviewed the manuscript. All authors read and approved the final manuscript. 


\section{Competing interests}

The authors declare that they have no competing interests.

\section{Consent for publication}

Not applicable.

\section{Ethics approval and consent to participate}

Not applicable.

\begin{abstract}
Author details
1 School of Informatics and Computing, Indiana University Bloomington, IN 47405, USA, Waterloo Road, 47405 Bloomington, IN, USA. ²Department of Computer Science and Engineering, Seoul National University, Seoul, Republic of Korea. ${ }^{3}$ Indiana University School of Medicine, Department of Cellular and Integrative Physiology, Medical Sciences Program, Bloomington, USA. ${ }^{4}$ Interdisciplinary Program in Bioinformatics, Seoul National University, Seoul, Republic of Korea. ${ }^{5}$ Bioinformatics Institute, Seoul National University, Seoul, Republic of Korea.
\end{abstract}

\section{Published: 23 December 2016}

\section{References}

1. Schnitt SJ. Classification and prognosis of invasive breast cancer: from morphology to molecular taxonomy. Mod Pathol. 2010;23:60-4.

2. Sorlie T, Perou CM, Tibshirani R, Aas T, Geisler S, Johnsen H, Hastie T, Eisen M, Van de Rijn M, Jeffrey S, et al. Gene expression patterns of breast carcinomas distinguish tumor subclasses with clinical implications. Proc Natl Acad Sci USA. 2001;98(19):10869-74.

3. Rivenbark AG, O'Connor SM, Coleman WB. Molecular and cellular heterogeneity in breast cancer: challenges for personalized medicine. Am J Pathol. 2013;183(4):1113-24.

4. Parker JS, Mullins M, Cheang MC, Leung S, Voduc D, Vickery T, Davies S, Fauron C, He X, Hu Z, et al. Supervised risk predictor of breast cancer based on intrinsic subtypes. J Clin Oncol. 2009;27(8):1160-7.

5. Hornberger J, Cosler LE, Lyman GH, et al. Economic analysis of targeting chemotherapy using a 21-gene RT-PCR assay in lymph-node-negative, estrogen-receptor-positive, early-stage breast cancer. Am J Manag Care. 2005; 11(5):313-24.

6. Gascard P, Bilenky M, Sigaroudinia M, Zhao J, Li L, Carles A, Delaney A, Tam A, Kamoh B, Cho S, et al. Epigenetic and transcriptional determinants of the human breast. Nat Commun. 2015;6:6351.

7. Smith AK, Conneely KN, Pace TW, Mister D, Felger JC, Kilaru V, Akel MJ, Vertino PM, Miller AH, Torres MA. Epigenetic changes associated with inflammation in breast cancer patients treated with chemotherapy. Brain Behav Immun. 2014;38:227-36.

8. Szyf M. DNA methylation signatures for breast cancer classification and prognosis. Genome Med. 2012;4(3):26.

9. Holm K, Hegardt C, Staaf J, Vallon-Christersson J, Jönsson G, Olsson H, Borg $\AA$, Ringnér $M$. Research article molecular subtypes of breast cancer are associated with characteristic DNA methylation patterns. Breast Cancer Res. 2010;12:R36.

10. Bediaga NG, Acha-Sagredo A, Guerra I, Viguri A, Albaina C, Ruiz Diaz I, Rezola R, Alberdi MJ, Dopazo J, Montaner D, et al. Dna methylation epigenotypes in breast cancer molecular subtypes. Breast Cancer Res. 2010;12(5):77.

11. Network CGA, et al. Comprehensive molecular portraits of human breast tumours. Nature. 2012;490(7418):61-70.

12. Conway K, Edmiston SN, May R, Kuan P, Chu H, Bryant C, Tse CK, Swift-Scanlan T, Geradts J, Troester MA, et al. Dna methylation profiling in the carolina breast cancer study defines cancer subclasses differing in clinicopathologic characteristics and survival. Breast Cancer Res. 2014;16(5):450.

13. Stefansson OA, Moran S, Gomez A, Sayols S, Arribas-Jorba C, Sandoval J, Hilmarsdottir H, Olafsdottir E, Tryggvadottir L, Jonasson JG, et al. A dna methylation-based definition of biologically distinct breast cancer subtypes. Mol Oncol. 2015;9(3):555-68.

14. Stirzaker C, Zotenko E, Song JZ, Qu W, Nair SS, Locke WJ, Stone A, Armstong NJ, Robinson MD, Dobrovic A, et al. Methylome sequencing in triple-negative breast cancer reveals distinct methylation clusters with prognostic value. Nat Commun. 2015;6:5899.
15. Fleischer $T$, Frigessi A, Johnson KC, Edvardsen $H$, Touleimat $N$, Klajic J, Riis ML, Haakensen VD, Wärnberg F, Naume B, et al. Genome-wide DNA methylation profiles in progression to in situ and invasive carcinoma of the breast with impact on gene transcription and prognosis. Genome Biol. 2014;15(8):435-2333349012841587.

16. Doi A, Park IH, Wen B, Murakami P, Aryee MJ, Irizarry R, Herb B, Ladd-Acosta C, Rho J, Loewer S, et al. Differential methylation of tissue-and cancer-specific CpG island shores distinguishes human induced pluripotent stem cells, embryonic stem cells and fibroblasts. Nat Genet. 2009;41(12):1350-3.

17. Brenet F, Moh M, Funk P, Feierstein E, Viale AJ, Socci ND, Scandura JM. DNA methylation of the first exon is tightly linked to transcriptional silencing. PloS one. 2011;6(1):14524.

18. Sproul D, Kitchen RR, Nestor CE, Dixon JM, Sims AH, Harrison DJ, Ramsahoye $\mathrm{BH}$, Meehan RR. Tissue of origin determines cancer-associated $\mathrm{CpG}$ island promoter hypermethylation patterns. Genome Biol. 2012;13(10):84.

19. Rhee JK, Kim K, Chae H, Evans J, Yan P, Zhang BT, Gray J, Spellman P, Huang TH-M, Nephew KP, et al. Integrated analysis of genome-wide DNA methylation and gene expression profiles in molecular subtypes of breast cancer. Nucleic Acids Res. 2013;41(18):8464-74.

20. Rao X, Evans J, Chae H, Pilrose J, Kim S, Yan P, Huang R, Lai H, Lin H, Liu Y, et al. CpG island shore methylation regulates caveolin-1 expression in breast cancer. Oncogene. 2013;32(38):4519-28.

21. Alexandrov LB, Nik-Zainal S, Wedge DC, Aparicio SA, Behjati S, Biankin $A V$, Bignell GR, Bolli N, Borg A, Børresen-Dale AL, et al. Signatures of mutational processes in human cancer. Nature. 2013;500(7463):415-21.

22. Watson IR, Takahashi K, Futreal PA, Chin L. Emerging patterns of somatic mutations in cancer. Nat Rev Genet. 2013;14(10):703-18.

23. Nik-Zainal S, Alexandrov LB, Wedge DC, Van Loo P, Greenman CD, Raine K, Jones D, Hinton J, Marshall J, Stebbings LA, et al. Mutational processes molding the genomes of 21 breast cancers. Cell. 2012;149(5):979-93.

24. Chuang TJ, Chen FC, Chen YZ. Position-dependent correlations between DNA methylation and the evolutionary rates of mammalian coding exons. Proc Natl Acad Sci. 2012;109(39):15841-6.

25. Xia J, Han L, Zhao Z. Investigating the relationship of DNA methylation with mutation rate and allele frequency in the human genome. BMC Genomics. 2012;13(Suppl 8):7.

26. Kim S, Li M, Paik H, Nephew K, Shi H, Kramer R, Xu D, Huang TH. Predicting DNA methylation susceptibility using CpG flanking sequences. Pac Symp Biocomput. 2008;315-26.

27. Yang $Y$, Nephew K, Kim S. A novel k-mer mixture logistic regression for methylation susceptibility modeling of cpg dinucleotides in human gene promoters. BMC Bioinform. 2012;13(3):1.

28. An J, Kim K, Rhee SM, Chae H, Nephew KP, Kim S. Genome-wide analysis and modeling of dna methylation susceptibility in 30 breast cancer cell lines by using cpg flanking sequences. J Bioinforma Comput Biol. 2013;11(03):1341003.

29. Chae H, Park J, Lee SW, Nephew KP, Kim S. Comparative analysis using mer and k-flank patterns provides evidence for cpg island sequence evolution in mammalian genomes. Nucleic Acids Res. 2013;41:4783-91.

30. Keshet I, Schlesinger Y, Farkash S, Rand E, Hecht M, Segal E, Pikarski E, Young RA, Niveleau A, Cedar $\mathrm{H}$, et al. Evidence for an instructive mechanism of de novo methylation in cancer cells. Nat Genet. 2006;38(2): 149-53.

31. Lee SM, Kim-Ha J, Choi WY, Lee J, Kim D, Lee J, Choi E, Kim YJ. Interplay of genetic and epigenetic alterations in hepatocellular carcinoma. Epigenomics. 2016;8(7):993-1005.

32. Krueger F. Trim galore. A wrapper tool around Cutadapt and FastQC to consistently apply quality and adapter trimming to FastQ files. 2015 http://www.bioinformatics.babraham.ac.uk/projects/trim_galore/. Accessed 16 Nov 2016.

33. Langmead B, Salzberg SL. Fast gapped-read alignment with bowtie 2 . Nat Methods. 2012;9(4):357-9.

34. Lienhard M, Grimm C, Morkel M, Herwig R, Chavez L. MEDIPS: genome-wide differential coverage analysis of sequencing data derived from DNA enrichment experiments. Bioinformatics. 2014;30(2):284-6.

35. Neve RM, Chin K, Fridlyand J, Yeh J, Baehner FL, Fevr T, Clark L, Bayani N, Coppe JP, Tong F, et al. A collection of breast cancer cell lines for the study of functionally distinct cancer subtypes. Cancer cell. 2006;10(6):515-27. 
36. Smyth GK. Limma: linear models for microarray data In: Gentleman $R$, Care V, Dudoit S, Irizarry R, Huber W, editors. Bioinformatics and computational biology solutions using $R$ and Bioconductor. New York: Springer; 2005. p. 397-420.

37. An J, Kim K, Chae H, Kim S. Degpack: A web package using a non-parametric and information theoretic algorithm to identify differentially expressed genes in multiclass rna-seq samples. Methods. 2014;69(3):306-14.

38. Akalin A, Kormaksson M, Li S, Garrett-Bakelman FE, Figueroa ME, Melnick A, Mason CE, et al. methylKit: a comprehensive R package for the analysis of genome-wide DNA methylation profiles. Genome Biol. 2012;13(10):87.

39. Krueger F, Andrews SR. Bismark: a flexible aligner and methylation caller for Bisulfite-Seq applications. Bioinformatics. 2011;27(11):1571-2.

40. Matys V, Fricke E, Geffers R, Gößling E, Haubrock M, Hehl R, Hornischer K, Karas D, Kel AE, Kel-Margoulis OV, et al. TRANSFAC ${ }^{\bullet}$ transcriptional regulation, from patterns to profiles. Nucleic Acids Res. 2003;31 (1):374-8.

41. Bovolenta LA, Acencio ML, Lemke N. HTRIdb: an open-access database for experimentally verified human transcriptional regulation interactions. BMC Genomics. 2012;13(1):1-10.

42. Lachmann A, Xu H, Krishnan J, Berger SI, Mazloom AR, Ma'ayan A. ChEA: transcription factor regulation inferred from integrating genome-wide ChIP-X experiments. Bioinformatics. 2010;26(19):2438-44.

43. Li H. A statistical framework for SNP calling, mutation discovery, association mapping and population genetical parameter estimation from sequencing data. Bioinformatics. 2011;27(21):2987-93.

44. Li H. Improving snp discovery by base alignment quality. Bioinformatics. 2011;27(8):1157-8.

45. Low JY, Nicholson HD. Epigenetic modifications of caveolae associated proteins in health and disease. BMC Genet. 2015;16(1):71.

46. Jadhav RR, Ye Z, Huang RL, Liu J, Hsu PY, Huang YW, Rangel LB, Lai HC, Roa JC, Kirma NB, Huang TH, Jin VX. Genome-wide DNA methylation analysis reveals estrogen-mediated epigenetic repression of metallothionein-1 gene cluster in breast cancer. Clin Epigenetics. 2015;7(1):13.

47. Kamalakaran S, Varadan V, Russnes HEG, Levy D, Kendall J, Janevski A, Riggs M, Banerjee N, Synnestvedt M, Schlichting E, et al. DNA methylation patterns in luminal breast cancers differ from non-luminal subtypes and can identify relapse risk independent of other clinical variables. Mol Oncol. 2011;5(1):77-92.

48. Yang X, Han H, De Carvalho DD, Lay FD, Jones PA, Liang G. Gene body methylation can alter gene expression and is a therapeutic target in cancer. Cancer Cell. 2014;26(4):577-90.

49. Hon GC, Hawkins RD, Caballero OL, Lo C, Lister R, Pelizzola M, Valsesia A, Ye Z, Kuan S, Edsall LE, et al. Global dna hypomethylation coupled to repressive chromatin domain formation and gene silencing in breast cancer. Genome research. 2012;22(2):246-58.

50. Schilling-Tóth B, Sándor N, Walter F, Bocsik A, Sáfrány G, Hegyesi H. Role of GDF15 in radiosensitivity of breast cancer cells. Open Life Sci. 2014;9(10):982-92.

51. Pan $X$, Chen Z, Huang R, Yao Y, Ma G. Transforming growth factor $\beta 1$ induces the expression of collagen type I by DNA methylation in cardiac fibroblasts. PloS one. 2013;8:e60335.

52. Lasabova Z, Tilandyova P, Kajo K, Zubor P, Burjanivova T, Danko J, Plank L. Hypermethylation of the GSTP1 promoter region in breast cancer is associated with prognostic clinicopathological parameters. Neoplasma. 2010;57(1):35

53. Goicoechea S, Bednarski B, Garcia-Mata R, Prentice-Dunn H, Kim H, Otey C. Palladin contributes to invasive motility in human breast cancer cells. Oncogene. 2009;28(4):587-98.

54. Chin YR, Toker A. The actin-bundling protein palladin is an Akt1-specific substrate that regulates breast cancer cell migration. Mol cell. 2010;38(3): 333-44.

55. Mugal CF, Ellegren H. Substitution rate variation at human $\mathrm{CpG}$ sites correlates with non- $\mathrm{CpG}$ divergence, methylation level and GC content. Genome Biol. 2011;12(6):58.

\section{Submit your next manuscript to BioMed Central and we will help you at every step:}

- We accept pre-submission inquiries

- Our selector tool helps you to find the most relevant journal

- We provide round the clock customer support

- Convenient online submission

- Thorough peer review

- Inclusion in PubMed and all major indexing services

- Maximum visibility for your research

Submit your manuscript at www.biomedcentral.com/submit

Biomed Central 